\title{
Reply to Dr. B. Karmazyn regarding 'Duodenum between the aorta and the SMA does not exclude malrotation'
}

\author{
Renaud Menten • Dana Dumitriu • Philippe Clapuyt • \\ David K. Yousefzadeh
}

Received: 12 October 2012 / Accepted: 18 October 2012 /Published online: 10 November 2012

(C) Springer-Verlag Berlin Heidelberg 2012

Sir, In response to Dr. Karmazyn's letter [1], we would like to clarify the following:

We disagree with the statement that the study evaluated patients with suspected malrotation, since this was not the case. The population, as stated in the materials and methods segment of the text, included asymptomatic patients explored for recurring respiratory symptoms [2]. Moreover, in all cases, US demonstrated the absence of whirlpool and the parallel position of the mesenteric vessels, excluding (unexpected) midgut volvulus.

We agree with the title of Dr. Karmazyn's letter, given that "the presence of the duodenum between the SMA and the aorta does not exclude malrotation," complicated by midgut volvulus. If the duodenum twists around the axis of the mesenteric vessels, as it does in midgut volvulus, this clockwise rotation can position a portion of it behind the mesenteric vessels, as shown in Figs. 3 and 4 in his letter [1]. However, in these cases, the authors did not show parallel orientation of the superior mesenteric vessels.

The embryology of intestinal rotation demonstrates the importance of the relationship between the duodenum and the mesenteric vessels. Upper gastrointestinal fluoroscopy defines the normal duodenal position in relation to bone structures. The advantage of US therefore resides in its ability to visualize the direct relationship between the mesenteric vessels and the duodenum and to identify those situations in particular in which the duodenum is not located in the aorto-mesenteric angle. In conclusion, retromesenteric D3 excludes uncomplicated malrotation, and parallel orientation of the mesenteric vessels excludes midgut volvulus. Only cross-sectional imaging as a whole and US in particular can achieve both objectives in the same setting and in the same study, not UGI.

\section{References}

1. Karmazyn B (2012) Duodenum between the aorta and the SMA does not exclude malrotation. Pediatr Radiol. doi:10.1007/ s00247-012-2537-5

2. Menten R, Reding R, Godding V et al (2012) Sonographic assessment of the retroperitoneal position of the third portion of the duodenum: an indicator of normal intestinal rotation. Pediatr Radiol 42:941-945
R. Menten $(\bowtie) \cdot$ D. Dumitriu $\cdot$ P. Clapuyt

Paediatric Radiology Unit, Cliniques Universitaires St. Luc,

Avenue Hippocrate 10,

Brussels 1200, Belgium

e-mail: renaud.menten@rdgn.ucl.ac.be

D. K. Yousefzadeh

Department of Radiology, University of Chicago,

Chicago, IL, USA 